\title{
Etude de l'influence des modes de transformation sur les teneurs en lycopène de quatre variétés de tomates de la région du nord du Burkina Faso
}

\author{
Ignace SAWADOGO ${ }^{1 *}$, Moumouni KOALA ${ }^{2}$, Constantin DABIRE ${ }^{2}$ \\ Lamousssa Paul OUATTARA ${ }^{1}$, Valérie B.E.J.T. BAZIE ${ }^{1,3}$, Adama HEMA ${ }^{2}$, \\ Charlemagne GNOULA ${ }^{3,4}$, Eloi PALE ${ }^{2}$ et Roger H.C. NEBIE $^{1}$ \\ ${ }^{I}$ Département Substances Naturelles/IRSAT/CNRST, 03 BP 7047 Ouagadougou 03, Burkina Faso. \\ ${ }^{2}$ Laboratoire de Chimie Organique et de Physique Appliqués, Université de Ouagadougou, \\ 03 BP 7021, Burkina Faso. \\ ${ }^{3}$ Centre de Recherche Biomoléculaire Pietro Annigoni (CERBA), Laboratoire de Biologie et Génétique \\ (LABIOGENE), Centre Médical Saint Camille, Ouagadougou, Burkina Faso. \\ ${ }^{4}$ Laboratoire de Pharmacologie, de Toxicologie et de Chimie Thérapeutique, Unité de Formation et de \\ Recherche en Sciences de la Sante' (UFR-SDS), Université de Ouagadougou, Burkina Faso. \\ *Auteur correspondant, E-mail: sawaigna@yahoo.fr ; Tel :00226 76563403
}

\section{RESUME}

Les traitements thermiques visent à stabiliser les aliments pour de longues périodes de stockage. Ils induisent des variations dans la composition de l'aliment. Le présent travail a pour but d'évaluer l'influence des modes de transformation (séchage, cuisson) sur les teneurs en lycopène de quatre variétés de tomates (Mongal F1, Tropimech, Royale et Rio Grande) de la région nord du Burkina Faso. Les résultats en milligrammes de lycopène par gramme de tomates sont ainsi exprimés : Purée : Tropimech $(0,065)$, Mongal F1 $(0,028)$, Royale $(0,051)$ et Rio Grande $(0,045$. Après les traitements on a noté les évolutions suivantes : Séchage à l'ombre: Tropimech (- 0.009), Mongal F1 (+0.009) Royale (-0.010) et Rio Grande (-0.009). Séchage solaire : Tropimech (-0.021), Mongal F1 (-0.007) Royale (-0.011) et Rio Grande (-0.014). Cuisson : Tropimech (-0.038), Mongal F1 (-0.014), Royale (-0.025) et Rio Grande (-0.024). Séchage solaire puis cuisson: Tropimech (-0.044), Mongal F1 (-0.017), Royale (-0.038) et Rio Grande (-0.0035). Séchage ombre puis cuisson: Tropimech (-0.045), Mongal F1 (-0.018), Royale (-0.035) et Rio Grande (-0.033). D'une manière générale, les teneurs en lycopène ont subi une baisse au cours de la transformation.

(C) 2015 International Formulae Group. All rights reserved.

Mots clés : Tomates, lycopène, mode de transformation.

\section{INTRODUCTION}

La tomate (Lycopersicon esculentum L.) est l'un des légumes le plus consommé principalement pour son apport en provitamine $\mathrm{A}$ sous forme de terpènes caroténoïdiens (Boumendjel et al., 2012). C'est un produit agricole riche en éléments nutritifs, notamment en lycopène, caroténoïde prédominant de la tomate avec un taux de 80\% (Rajoria et al., 2010). Principal colorant responsable de la couleur rougefoncé caractéristique des fruits mûrs de la tomate, le lycopène a suscité beaucoup d'attention ces dernières années en raison de 
son effet bénéfique dans la prévention de certaines pathologies (Markovic et al., 2006).

Des études épidémiologiques ont en effet montré que la consommation de tomates et des produits à base de tomates pourraient prévenir certaines maladies chroniques tels que les cancers de la cavité buccale, du pharynx, de l'œsophage, de l'estomac, du rectum, de la prostate et du sein (Vaishampayan et al., 2007, Ferreira et al., 2000) ainsi que les risques de maladies cardiovasculaires (Riccioni, 2009).

La production de tomates en 2010 au Burkina Faso était estimée à 157086 tonnes, soit $21 \%$ de la production maraichère totale. La région du Nord détient la plus grande production avec 39639 tonnes, soit $25 \%$ de la production totale de tomate selon le Recensement Général de l'Agriculture (RGA, 2006-2010). Plusieurs variétés de tomates y sont cultivées dont les principales sont : Tropimech, Mongal F1, Royale et Rio Grande.

Les procédés de transformation et de préparation impliquent le plus souvent des traitements thermiques susceptibles d'affecter les teneurs en micronutriments et partant, la qualité nutritionnelle des produits finis.

Pour ces variétés, très peu d'études se sont intéressées à l'évaluation des teneurs en lycopène et à l'influence des traitements thermiques sur le lycopène, d'où l'intérêt de cette étude dont les objectifs sont d'une part de déterminer les teneurs en lycopène des variétés de tomates les plus cultivées dans la région du Nord et d'autre part de mettre en évidence l'influence des modes de transformation sur ces teneurs.

\section{MATERIEL ET METHODES \\ Matériel végétal}

Les quatre variétés de tomates (Tropimech, Royale, Rio Grande, Mongal F1) à maturité ont été récoltées dans les champs auprès des producteurs de Yako (Komtoega) et Ouahigouya (Goinré). Les échantillons de tomates ont été gardés soit à l'état frais, soit à l'état sec pour le dosage des teneurs en lycopène. Les échantillons secs ont été obtenus par séchage naturel à l'ombre au laboratoire pendant sept (07) jours, soit à l'aide d'un séchoir solaire pendant quatre (04) jours. La tomate séchée a ensuite été broyée à l'aide d'un mixeur de type SEVEN 7 STAR.

\section{Méthode}

La teneur en lycopène est déterminée par la méthode spectrophotométrique directe telle que décrite par Fish et al. (2002) avec de légères modifications. Une courbe d'étalonnage a d'abord été établie à partir de différentes concentrations de solutions de lycopène allant de 0,00782 à $0,125 \mathrm{mg} / \mathrm{ml}$.

Les extraits analysés ont été obtenus à partir de la tomate fraîche (purée), tomate séchée (à l'ombre ou à l'aide du séchoir solaire) et la tomate cuite (sauce tomate préparée soit à partir de la purée ou à partir de la tomate séchée) selon la méthode de Chanforan (2010).

\section{Préparation des extraits}

Pour la préparation des extraits, $1,5 \mathrm{~g}$ de chaque échantillon ont été extraits avec 20 $\mathrm{ml}$ de système de solvant hexane - acétone méthanol (50:25:25) stabilisé avec $0.05 \%$ de Butylhydroxytoluène (BHT) par macération sous agitation magnétique pendant quinze minutes ( $15 \mathrm{mn}$ ). Puis $5 \mathrm{ml}$ d'eau distillée ont été ajoutées; la solution a été agitée de nouveau pendant 5 minutes et après décantation, la phase organique a été recueillie pour le dosage. L'opération a été ainsi répétée trois fois.

\section{Dosage spectrophotométrique}

L'absorbance de $0,5 \mathrm{ml}$ de la phase organique, convenablement diluée a été mesurée à $502 \mathrm{~nm}$ à l'aide d'un spectrophotomètre de type CIBA-corning 2800 Spectrascan.

La teneur en lycopène, exprimée en milligrammes par gramme $(\mathrm{mg} / \mathrm{g})$ de tomates est donnée par la formule suivante (Fish et al., 2002 ; Kong et Ismail, 2011) : 


\section{C}

$$
\text { lyc }(\mathrm{mg} / \mathrm{g})=\frac{\mathrm{Abs502} \cdot \mathrm{Fd} \cdot \mathrm{Mlyc}_{\mathrm{lyd}}}{\varepsilon_{\mathrm{Lyc}(502) \cdot \mathrm{mech}}}
$$

$\mathrm{C}_{\text {lyc : }}$ Concentration de lycopène en milligramme par gramme, Abs502: Absorbance à $502 \mathrm{~nm}, \mathrm{Fd}$ : facteur de dilution, mech: masse de l'échantillon, $\mathrm{Vd}$ : volume d'extraction, $\mathbf{M}_{\mathrm{lyc}}$ : Masse molaire $\mathrm{du}$ lycopène, ${ }^{\varepsilon}$ Lyc: coefficient d'extinction molaire du lycopène.

\section{Analyses statistiques}

Les données des analyses spectrophotométriques ont été traitées avec les logiciels Excel 2010 et GenStat Release 14.0 (PC/Windows 7). Les tests de dosage ont été répétés en triple par échantillon. Les résultats ont été exprimés sous la forme moyenne \pm écart type. Les valeurs de $\mathrm{p}<0,05$ sont considérées statistiquement significatives.

\section{RESULTATS}

Les résultats de l'ensemble des analyses ont été consignés dans le Tableau 1. Ils sont exprimés en milligrammes par gramme de tomates $(\mathrm{mg} / \mathrm{g})$.

Teneur en lycopène dans la purée des quatre (4) variétés de tomates

Les résultats de l'analyse de la purée des quatre variétés de tomates ont révélé que Tropimech possède la plus forte teneur en lycopène $(0,065 \mathrm{mg} / \mathrm{g})$. On observe la plus faible valeur avec Mongal F1 (0,28 mg/g). Rio Grande et Royale ont respectivement 0,045 et $0,051 \mathrm{mg} / \mathrm{g}$. Les résultats sont représentés par la Figure 1.

\section{Effet du séchage à l'ombre sur la teneur en lycopène}

La Figure 2 établit une comparaison entre les teneurs en lycopène de la purée de tomates obtenue par broyage de la tomate fraîche et celles des tomates séchées à l'ombre. Ces résultats montrent une diminution des teneurs en lycopène des variétés Tropimech $(0,065-0,056)$, Rio Grande $(0,045-0,035)$ et Royale $(0,05-0,041)$ par rapport à la purée de tomates et une augmentation au niveau de Mongal F1 (0,0280,037). Soit une diminution de $13,8 \%$ pour Tropimech, Royale $(19,6 \%)$ et Rio Grande $(17,8 \%)$. Mongal F1 a subit une hausse de $25 \%$.

\section{Effet du séchage solaire sur la teneur en lycopène}

$\mathrm{Au}$ cours du séchage solaire, on relève une diminution des teneurs au niveau de toutes les variétés mais à des degrés divers. Mongal F1 (0,028-0,021), Tropimech (0,065$0,044)$, Rio Grande $(0,045-0,031)$ et Royale (0,05-0,039). La baisse est beaucoup plus sensible pour la variété Rio Grande avec une perte de $31,1 \%$. Mongal F1 a subi une baisse de $15 \%$, Tropimech perd $30,7 \%$ tandis que Royale régresse de 23,5\%. La Figure 3 établit une comparaison entre les teneurs en lycopène de la purée de tomates fraîches et celles obtenues après séchage solaire.

\section{Effet de la cuisson de la purée de tomates sur la teneur en lycopène}

On note au cours de la cuisson de la purée de tomates une diminution des teneurs au niveau de toutes les variétés. Tropimech dont la valeur a varié de 0,065 à $0,027 \mathrm{mg} / \mathrm{g}$ perd $58,4 \%$ de sa teneur tandis que Royale $(0,05-0,026)$ perd $53,3 \%$, Rio Grande $(0,045$ $0,021)$ perd $49,02 \%$ et enfin Mongal F1 $(0,028-0,014)$ régresse de $50 \%$. La Figure 4 présente les teneurs en lycopène des purées de tomates et celles obtenues après cuisson.

\section{Effet du séchage à l'ombre et de la cuisson sur la teneur en lycopène}

La cuisson de la tomate séchée à l'ombre montre une sensibilité des différentes variétés de tomates à l'effet de cuisson se traduisant par une baisse des teneurs en lycopène. Tropimech (0,065-0,056-0,02), Mongal F1 (0,028-0,037-0,011), Royale $(0,05-0,041-0,015)$ et Rio Grande $(0,045$ 0,035-0,012). Les valeurs entre parenthèse représentent les teneurs des purées, des 
tomates séchées à l'ombre et celles des tomates séchées à l'ombre puis cuites.

De la purée à la cuisson après séchage à l'ombre, on note une baisse de 60,7 ; 69,2 ; 70,6 et $73,3 \%$ respectivement pour Mongal F1, Tropimech, Royale et Rio Grande. La Figure 5 compare les teneurs en lycopène des purées de tomates et celles obtenues après séchage à l'ombre puis cuites.

Effet du séchage solaire et de la cuisson sur la teneur en lycopène

On constate une diminution des teneurs en lycopène après cuisson des tomates séchées au soleil (Figure 6). Mongal F1
(0,028-0,021-0,011), Royale (0.05-0,0390,012), Tropimech (0,065-0,044-0,019) 0et Rio Grande (0,045-0,031-0,010). Les valeurs entre parenthèse représentent respectivement les teneurs de la purée de tomates, des tomates séchées au soleil et celles des tomates séchées au soleil puis cuites. Les valeurs des tomates séchées puis cuites ont subi une baisse de 60,$7 ; 70,8 ; 77,8$ et $76,5 \%$ respectivement pour Mongal F1, Tropimech, Royale et Rio Grande par rapport à celles de la purée.

Tableau 1 : Teneur en lycopène des variétés de tomates en fonction des différents traitements.

\begin{tabular}{lcccc}
\hline & Mongal F1 & Tropimech & Rio Grande & Royale \\
\hline LP & $0,028 \pm 0,001^{\mathrm{a}}$ & $0.065 \pm 0,0003^{\mathrm{b}}$ & $0,045 \pm 0,0003^{\mathrm{ab}}$ & $0,051 \pm 0,01^{\mathrm{b}}$ \\
LO & $0,035 \pm 0,001^{\mathrm{a}}$ & $0,056 \pm 0,003^{\mathrm{b}}$ & $0,037 \pm 0.0001^{\mathrm{a}}$ & $0,041 \pm 0.002^{\mathrm{a}}$ \\
LS & $0,021 \pm 0.0005^{\mathrm{a}}$ & $0,045 \pm 0.006^{\mathrm{b}}$ & $0,031 \pm 0.001^{\mathrm{ab}}$ & $0,039 \pm 0.005^{\mathrm{ab}}$ \\
LC & $0,014 \pm 0.002^{\mathrm{a}}$ & $0,027 \pm 0,01^{\mathrm{c}}$ & $0,021 \pm 0,001^{\mathrm{b}}$ & $0,026 \pm 0.004^{\mathrm{c}}$ \\
LOC $^{\mathrm{b}}$ & $0.011 \pm 0.004^{\mathrm{a}}$ & $0.02 \pm 0.002^{\mathrm{c}}$ & $0.012 \pm 0.008^{\mathrm{b}}$ & $0.015 \pm 0.009^{\mathrm{c}}$ \\
LSC & $0.011 \pm 0.0001^{\mathrm{a}}$ & $0.019 \pm 0.0002^{\mathrm{c}}$ & $0.010 \pm 0.0004^{\mathrm{a}}$ & $0.012 \pm 0.0001^{\mathrm{b}}$
\end{tabular}

LP : teneur en lycopène de la purée de tomate, LO : teneur en lycopène de la tomate séchée à l'ombre, LS : teneur en lycopène de la tomate séchée au soleil, LC : teneur en lycopène de la tomate cuite, LOC : teneur en lycopène de la tomate séchée à l'ombre puis cuite, LSC : teneur en lycopène de la tomate séchée au soleil puis cuite.

Les lettres différentes sur les valeurs signifient que ces valeurs sont statistiquement différentes

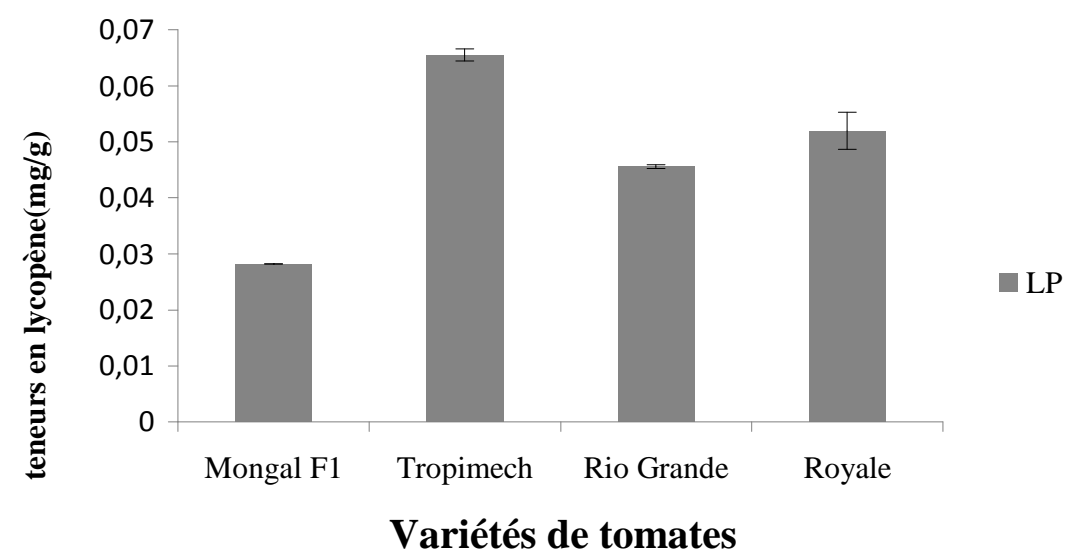

Figure 1 : Teneurs en lycopène en fonction des variétés de tomates fraîches. 


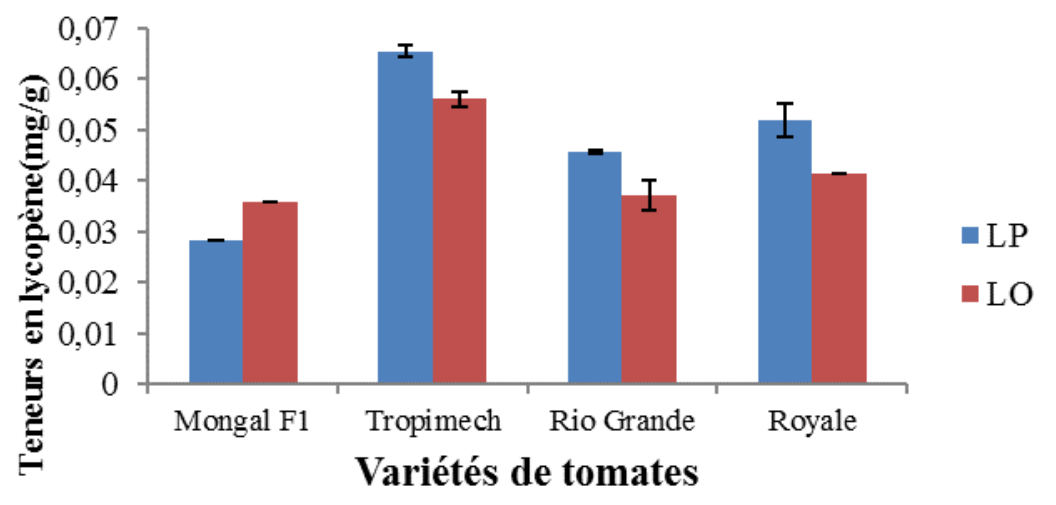

Figure 2: Effet du séchage à l'ombre sur la teneur en lycopène.

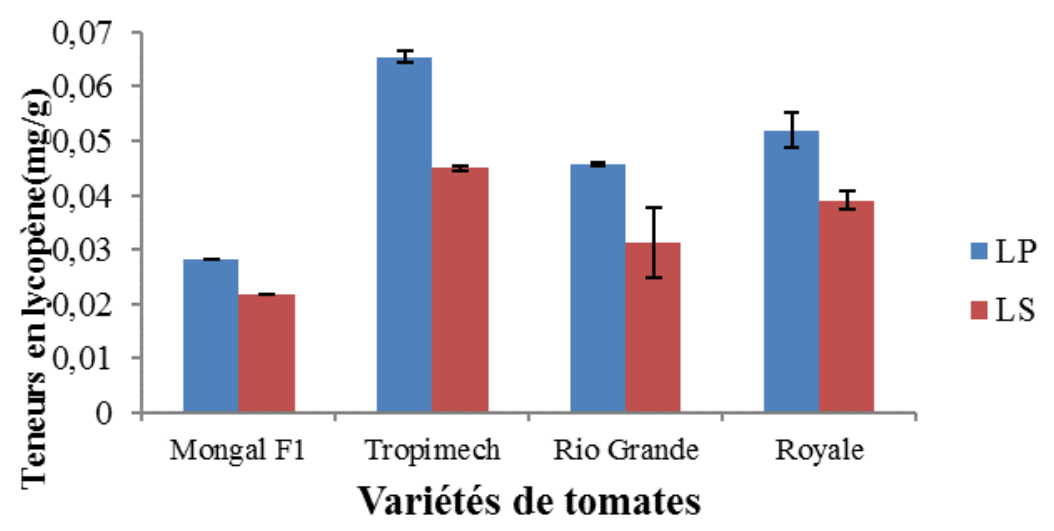

Figure 3 : Effet du séchage solaire sur la teneur en lycopène.

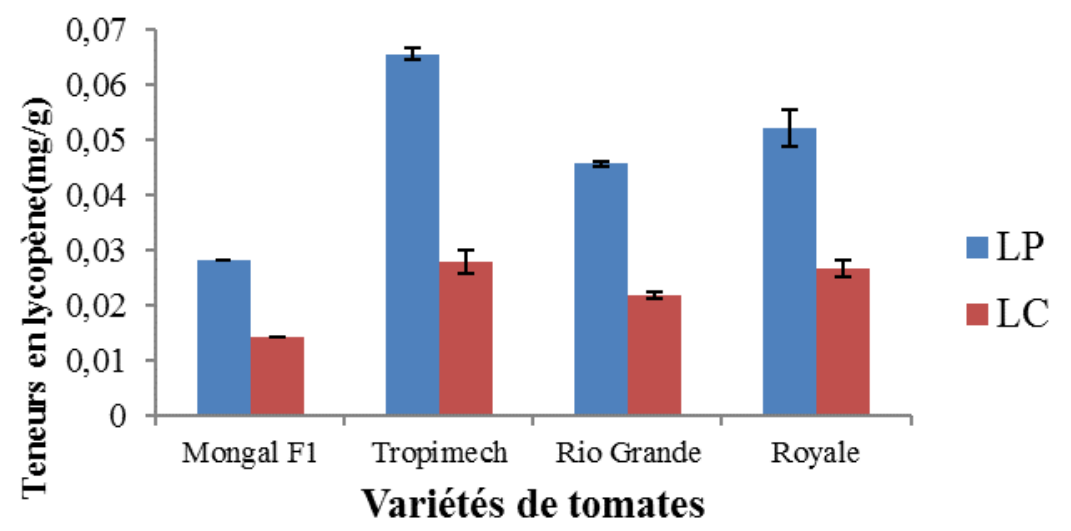

Figure 4 : Effet de la cuisson de la tomate fraîche sur la teneur en lycopène. 


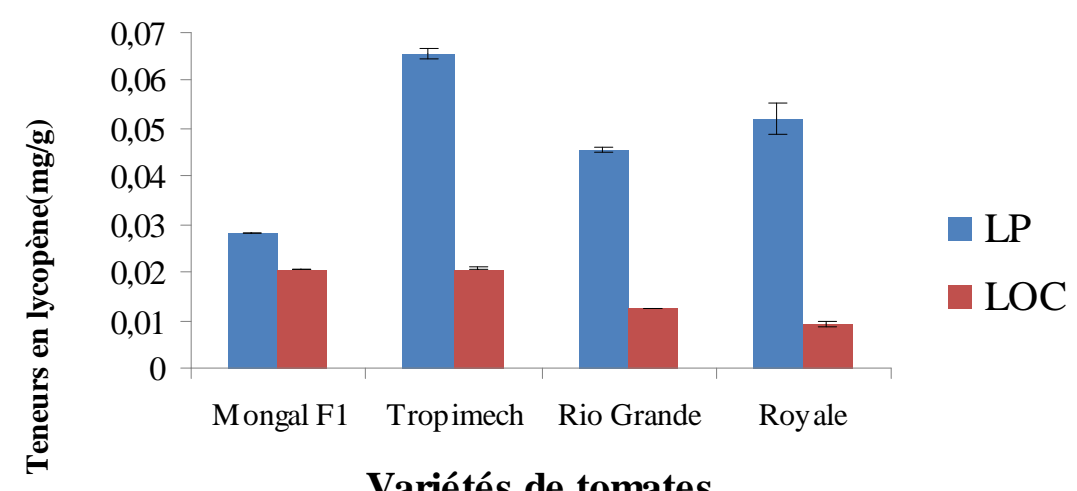

Figure 5 : Effet du séchage à l'ombre et de la cuisson sur la teneur en lycopène.

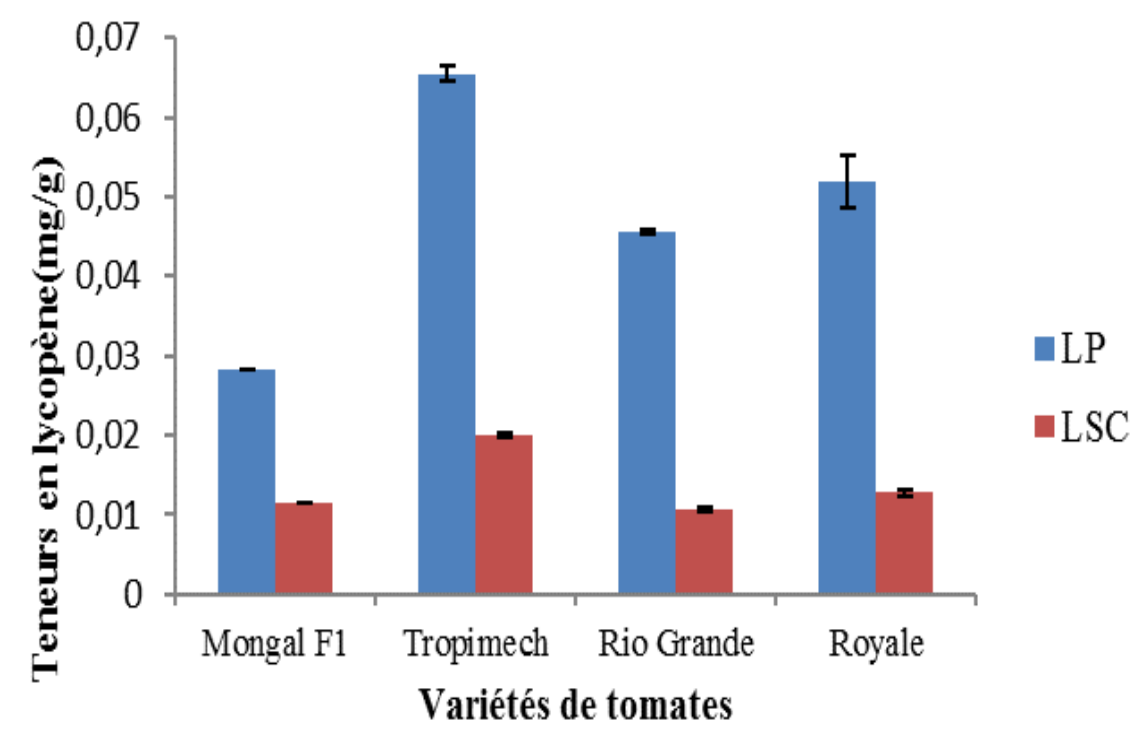

Figure 6: Effet du séchage solaire et de la cuisson sur la teneur en lycopène.

\section{DISCUSSION}

Les teneurs en lycopène de Tropimech $(0,065 \mathrm{mg} / \mathrm{g})$, Royale $(0,051 \mathrm{mg} / \mathrm{g})$, Rio Grande $(0,045 \mathrm{mg} / \mathrm{g})$ et Mongal F1 $(0,28$ $\mathrm{mg} / \mathrm{g}$ ) (Figure 1) ne montrent pas une grande différence avec celles contenues dans la littérature. En effet, Mendelová et al. (2013) ont évalué les teneurs en lycopène de la tomate fraîche de six variétés de tomates Darina F1, Denár, Kecskeméty, Orange,
Paulína F1 and Šejk F1) et ont trouvé des valeurs comprises entre 8,99 et $6,14 \mathrm{mg} / 100$ $\mathrm{g}$ de tomates fraîches. De même, Toor et al. (2006) ont trouvé sur trois variétés étudiées, des valeurs comprises entre 2,7 et $4,7 \mathrm{mg} / 100$ g. Ces différences de résultats viennent confirmer le fait que les teneurs en lycopène diffèrent d'une variété à une autre. D'autres facteurs pourraient également expliquer cette différence de teneurs. En effet, Helyes et al. 
(2007) ont montré que les facteurs environnementaux, telle qu'une température élevée de la surface du fruit provoquée par une température de l'air élevée ou une lumière $\mathrm{du}$ soleil directe, diminuent le contenu de lycopène dans la peau de fruit et diminuent également de manière significative la teneur en lycopène du fruit entier. Helyes et al. (2006) ont en outre montré que la teneur en lycopène augmente avec la maturation du fruit. Aussi, Chanforan (2010) a montré que les conditions de culture (date de récolte dans l'année, ensoleillement, température, qualité du sol, etc.) peuvent affecter grandement la teneur en caroténoïdes.

La diminution des teneurs des variétés Tropimech, Royale et Rio Grande au cours du séchage à l'ombre (Figure 2) montre que ce mode de traitement a une influence négative sur les teneurs en lycopène de certaines variétés. Les teneurs subissent une diminution de l'ordre de 13,6 à 19,6\%. Cette influence négative serait probablement due à la photosensibilité du lycopène. En effet, selon Kumar et al. (2014), la structure chimique du lycopène en particulier la longue chaîne conjuguée de doubles liaisons $\mathrm{C}=\mathrm{C}$, prédispose le lycopène à l'isomérisation et à la dégradation lors de l'exposition à la lumière et à la chaleur.

Par contre, l'augmentation de $25 \%$ de la teneur en lycopène de Mongal F1 trouverait son explication dans le fait que le séchage a entraîné une rupture des membranes cellulaires des parois et a libéré le lycopène emprisonné dans les matrices (Abushita et al., 2000).

Les résultats permettent également d'affirmer que la sensibilité au séchage à l'ombre est fonction de la variété de tomates.

Le séchage solaire affecte négativement les teneurs en lycopène de toutes les variétés de tomates. Les diminutions sont dans l'ordre de 15 à $31,1 \%$ en fonction de la variété (Figure 3). Cette baisse se justifierait par l'action double de la chaleur et de la lumière du soleil.

L'influence négative de la cuisson sur la composition en lycopène des différentes variétés de tomates s'est traduite par une baisse comprise entre de 50 et $58,4 \%$ de la teneur initiale (Figure 4). En effet, la haute température combinée avec le long temps d'exposition a montré un effet négatif sur des caroténoïdes (Dorais et al., 2008). L'ampleur de la diminution des teneurs varie en fonction des variétés.

La teneur en lycopène de la tomate séchée soumise à la cuisson (Figure 5) a subit une nette régression du fait de la conjugaison de plusieurs éléments notamment la lumière du jour, la chaleur et la température de cuisson. La teneur a considérablement diminué par rapport à la tomate séchée à l'ombre dans l'ordre de 51 à 85,72\% et de 61 à $78 \%$ par rapport à la purée.

La tomate séchée au soleil puis cuite a également subi une régression énorme par rapport aux tomates séchées uniquement au soleil et à la purée (Figure 6). La lumière solaire, la température et l'oxygène de l'air ont agi négativement sur le lycopène. Toute chose qui a entraîné une diminution de 61 à $78 \%$ par rapport à la purée et de 40 à $54 \%$ par rapport à la tomate séchée au soleil. Les microconstituants antioxydants principalement les caroténoïdes sensibles à la chaleur et à la lumière peuvent être partiellement dégradés lors de réactions d'oxydation et/ou d'isomérisation (Chanforan, 2010). Aussi, l'oxygène est susceptible de réagir avec des radicaux dérivés des caroténoïdes pour produire des radicaux peroxyles capables de propager la peroxydation lipidique. Ainsi, lors de la préparation des produits à base de tomates, la présence d'importante quantité d'oxygène peut avoir pour conséquence une diminution de la teneur en caroténoïdes. Cette réaction peut, par ailleurs, être accélérée par la 
température et la présence d'huile (Mayeaux et al., 2006, Sahlin et al.,2004).

\section{Conclusion}

La teneur en lycopène des tomates dépend des variétés. Cette étude a révélé que Tropimech est la variété qui a la teneur en lycopène la plus élevée. Il en ressort également que toutes les variétés de tomates à l'exception de Mongal F1 dont la teneur a connu une hausse au cours du séchage à l'ombre sont sensibles aux différents traitements. Cette sensibilité se traduit en règle générale par une diminution de teneurs. $\mathrm{Au}$ regard des résultats, il serait souhaitable que soit encouragé la consommation des tomates à l'état frais et que la culture des variétés, Tropimech, Royale et Rio grande soit encouragée et vulgarisée eu égard à leur teneur élevée en lycopène. Des investigations plus poussées doivent également être menées en vue d'une valorisation du lycopène des sous-produits des unités de transformation de tomates et de son incorporation dans l'alimentation humaine au regard de ses effets bénéfiques pour la santé.

\section{REFERENCES}

Abushita AA, Daood, HG, Biacs PA. 2000. Change in carotenoids and antioxidant vitamins in tomato as a function of varietal and technological factors. J. Agr. Food Chem., 48: 2075-2081.

Boumendjel MM, Houhamdi MF, Samar H, Sabeg A, Boutebba M. 2012. Effet des traitements thermiques d'appertisation sur la qualité biochimique, nutritionnelle et technologique du simple, double et triple concentre de tomate. Sciences et Technologies, 36: 51-59.

Chanforan C. 2010. Stabilité de microconstituants de la tomate (composés phénoliques, caroténoïdes, vitamines $\mathrm{C}$ et E) au cours des procédés de transformation : études en systèmes modèles, mise au point d'un modèle stoechio-cinétique et validation pour l'étape unitaire de préparation de sauce tomate. Thèse de Doctorat. Université d'Avignon et des pays de Vaucluse, p. 399.

Dorais M, Ehret DL, Papadopoulos AP. 2008. Tomato (Solanum lycopersicum) health components, from the seed to the consumer. Phytochem Rev., 7(2): 231250.

Ferreira AL, Yeum KJ, Liu C, Smith D, Krinsky NI, Wang XD, Russell RM. 2000. Tissue distribution of lycopene in ferrets and rats after lycopene supplementation. J. Nutr., 130(5): 12561260.

Fish WW, Perkins-Veazie P, Collins JK. 2002. A Quantitative Assay for Lycopene That Utilizes Reduced Volumes of Organic Solvents. J. Food Compos. Anal., 15(3): 309-317.

Helyes L, Lugasi A, Pék Z. 2007. Effect of natural light on surface temperature and lycopene content of vine ripened tomato fruit. Can. J. Plant Sci., 87: 927-929.

Helyes L, Zoltan pek, Andrea Lugasi. 2006. Tomato fruit quality and content depend on stage of maturity. Hortscience, 41(6): 1400-14001.

Kong KW, Ismail A. 2011. Lycopene content and lipophilic antioxidant capacity of by products from Psidium guajava fruits produced during puree production industry. Food Bioprod Process, 89(1): 53-61.

Kumar A, Bhawsar NG, Anghore K, Gayakwad A, Panse U, Gayakwad SR, Khasedo K, Patekar K, Nagale BD Lajras AD. 2014. Effect of temperature on quantities of lycopene in some replicate of tomatoes. IJPT, 4(3): 142-145.

Markovic K, Hruskar M, Vahcic N. 2006. Lycopene content of tomato products and 
their contribution to the lycopene intake of Croatians. Nutr. Res., 26(11): 556-560.

Mayeaux M, Xu Z, King,JM, Prinyawiwatkul W. 2006. Effects of cooking conditions on the lycopene content in tomatoes. $J$. Food Sci., 71: 461-464.

Mendelova A, Fikselova M, Mendel L. 2013. Carotenoids and lycopene content in fresh and dried tomato fruits and tomato juice. Acta Univ. Agric. Fac. Agron., 5: 13291337.

Rajoria A, KumarJ, Chauhan AK. 2010. Antioxidative and anticarcinogenic role of lycopene in human health. J. Dairy Foods Home Sci., 3(4): 157-165.

Riccioni G. 2009. Carotenoids and Cardiovascular Disease. Curr. Atheroscler Rep., 11: 434-439.
Sahlin E, Savage GP, Lister CE. 2004. Investigation of the antioxidant properties of tomatoes after processing. $J$. Food Compos. Anal., 17: 635-647.

Toor RK, Savage GP, Lister CE. 2006. Seasonal variations in the antioxidant composition of greenhouse grown tomatoes. J. Food Compos Anal., 19: 110.

Vaishampayan U, Hussain M, Banerjee M, Seren S, Sarkar FH, Fontana J, Forman JD, Cher ML, Powell I, Pontes JE, Kucuk O. 2007. Lycopene and soy isoflavones in the treatment of prostate cancer. Nutr. Cancer, 59(1): 1-7. 